\title{
Redescription de Quimperia lanceolata Gendre, 1926 (Nematoda, Seuratoidea)
}

\author{
par G. VASSILIADES \\ Laboratoire national de Recherches vétérinaires - Dakar (Sénégal) \\ et Institui d'Elevage et de Médecine vétérinaire des Pays tropicaux - F 94 - Maisons-Alfort
}

\begin{abstract}
Résumé
Redescription de Quimperia lanceolata Gendre, 1926, parasite habituel de Ctenopoma kingsleyae Günther, Poisson Anabantidae, du Sénégal. Des précisions morphologiques sont données concernant notamment la structure céphalique et les spicules; l'absence de gubernaculum est confirmée. C'est la deuxième fois seulement que l'espèce est signalée en Afrique.
\end{abstract}

\section{Summary}

Redescription of Quimperia lanceolata Gendre, 1926, usual parasite of Ctenopoma kingsleyae Günther, Anabantidae Fish of Senegal. Morphological precisions are given, especially concerning the cephalic structure and the spicules; the absence of gubernaculum is confirmed. This species is signalized in Africa for the second time.

En 1926, Gendre décrit une espèce et un genre nouveau à partir de deux femelles et un mâle découverts chez un «Poisson Acanthoptérygien non déterminé », capturé le 27 février 1908 en Guinée (Cercle de Labé). Il s'agit de Quimperia lanceolata, genre 
monospécifique classé provisoirement par son auteur dans les Nématodes «incertae sedis ».

En 1928, Gendre élève son nouveau genre au niveau d'une sous-famille, celle des Quimperiinae.

Chabaud, Campana-Rouget et Brygoo, 1959 et 1960, regroupent les Quimperiinae Gendre, 1928 avec d'autres sous-familles dans la famille des Seuratidae (Hall, 1916, sub. fam.), Chabaud, Campana-Rouget et Brygoo, 1959, dans l'ordre des Ascaridida.

Depuis la découverte de Gendre, personne à notre connaissance ne paraît avoir retrouvé cette espèce.

Récemment, nous avons eu l'occasion de rencontrer à plusieurs reprises chez des Ctenopoma pêchés dans les marigots de Sangalkam, près de Rufisque, des Nématodes que nous pensons pouvoir rattacher à Quimperia lanceolata.

Nous donnons ci-après la description de nos spécimens.

\section{Matériel étudié}

Hôte: Ctenopoma kingsleyae Günther, Famille des Anabantidae, sous-ordre des Anabantoidei, Téléostéens, Perciformes. Sénégal).

Lieu de RÉColte: Rivière de Sangalkam, Rufisque, (Région du Cap-Vert,

LOCALISATION DES PARASITES: intestin.

MATÉRIEl RÉCOlté : 12-4-1970 : trois mâles et une femelle, enregistrés au laboratoire d'Helminthologie du Laboratoire national de Recherches vétérinaires de Dakar sous le $\mathrm{n}^{\circ}$ A 57. Néotypes redécrits: un mâle et une femelle déposés dans les collections du Laboratoire de Zoologie (Vers) du Muséum national d'Histoire naturelle de Paris sous le $\mathrm{n}^{\circ} 104 \mathrm{BA}$.

\section{Description}

Ce sont de petits Nématodes très fins, aux extrémités très effilées, blanc nacré et transparents quand ils sont vivants.

La femelle est légèrement plus grande que le mâle.

La cuticule, très fine, est très légèrement striée transversalement et longitudinalement.

La présence d'ailes latérales antérieures, débutant un peu plus bas en arrière des lèvres et se terminant au niveau de deux petites déirides, en avant de la terminaison œsophagienne, donne au corps un aspect «lancéolé » (d'où le nom d'espèce donné par Gendre) (fig. 1, A).

Absence de cavité buccale. Bouche triangulaire bordée par trois petites lèvres globuleuses portant chacune sur leur bord libre, deux petites expansions coniques (\& boutons sensitifs » pour Gendre, 1926). 


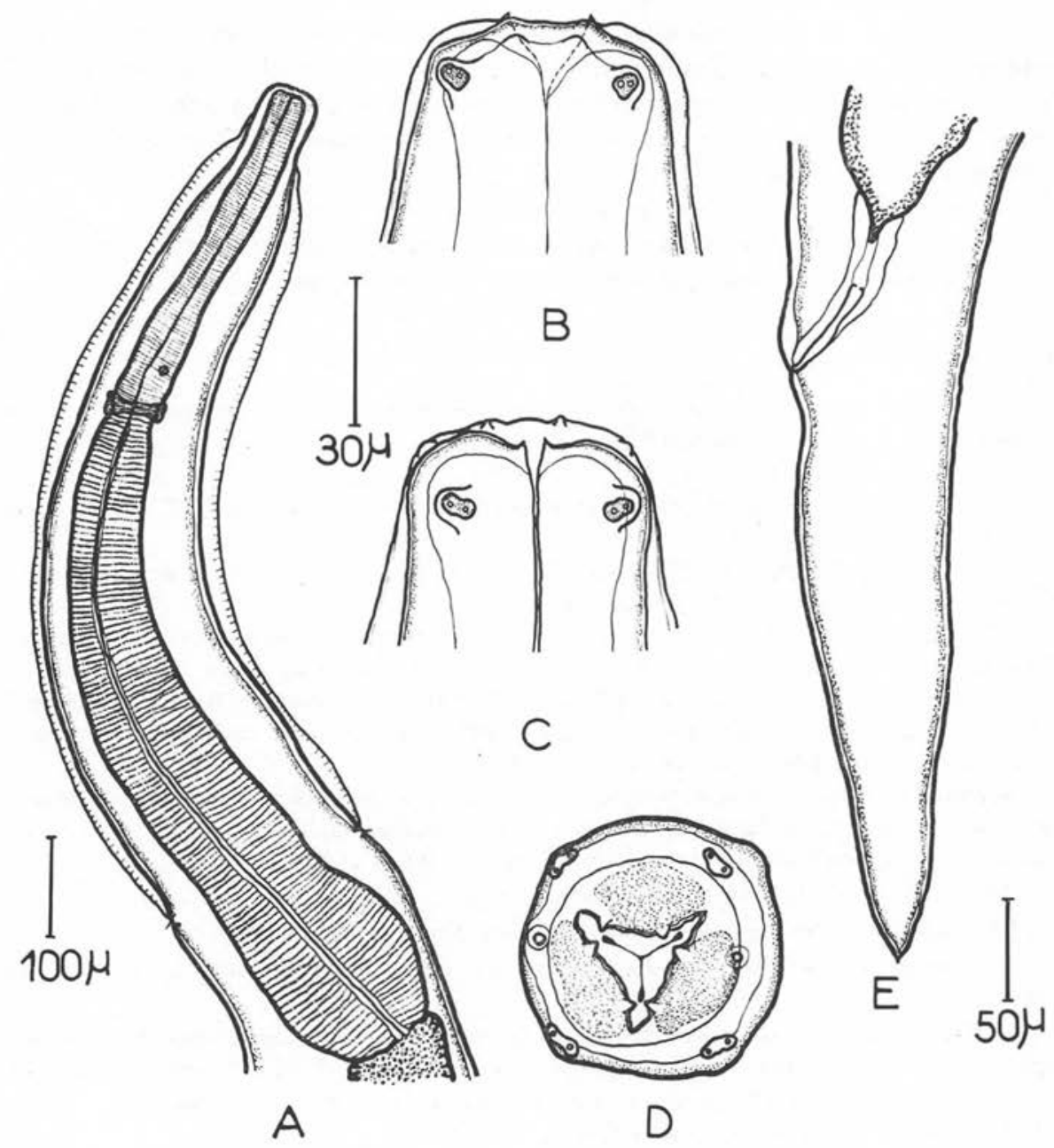

FIG. I. - Quimperia lanceolata Gendre, 1926
A : extrémité antérieure de la femelle, vue dorsale.
B : région buccale, vue dorsale.
C : région buccale, vue latérale.
$\mathrm{D}$ : région buccale, vue apicale.
E : queue de la femelle, vue latérale.

(A: échelle $100 \mu ; \mathrm{B}, \mathrm{C}, \mathrm{D}$ : échelle $30 \mu$; E : échelle $50 \mu$ ) 
Cycle externe de quatre grosses papilles submédianes probablement doubles; deux papilles sur la lèvre dorsale et une papille sur chacune des deux lèvres latéro-ventrales qui portent également chacune une amphide latérale (fig. 1, B, C, D).

L'œsophage est entièrement musculaire, mais revêt deux aspects différents. Il comprend une partie antérieure étroite et cylindrique jusqu'au niveau de l'anneau nerveux, puis, lui faisant suite, une partie postérieure plus longue, plus large et plus musculaire, de plus en plus large vers sa base bien dilatée sans constituer cependant un véritable bulbe (fig. $1, \mathrm{~A}$ ).

Le pore excréteur est situé au niveau de l'anneau nerveux.

Le mâle possède deux spicules égaux, mais pas de gubernaculum.

La femelle est didelphe, ovipare, à vulve post-équatoriale.

\section{Description du mâle.}

Corps long de $9,750 \mathrm{~mm}$ et large de $220 \mu$ au niveau de l'intestin antérieur. Toutes les dimensions données se rapportent à cet exemplaire.

Cuticule épaisse de 2 à $3 \mu$.

Pore excréteur, anneau nerveux et déirides respectivement situés à $375 \mu, 430 \mu$ et $950 \mu$ de l'extrémité antérieure.

Ailes latérales longues de $850 \mu$ sur $35 \mu$ de large, débutant à $50 \mu$ de l'apex et se terminant à $900 \mu$, au niveau des déirides.

Esophage antérieur long de $450 \mu$ sur $50 \mu$ de large; œsophage postérieur long de $650 \mu$ sur $120 \mu$ de largeur maximale. Longueur totale de l'œsophage égale à $1.100 \mu$.

Queue incurvée ventralement sur elle-même décrivant une spire simple et terminée par une pointe aiguë. Cuticule légèrement élargie à $300 \mu$ de la pointe caudale donnant naissance à des ailes caudales très étroites (fig. II, A, B, C).

Ventouse caudale faiblement musculaire, sans anneau chitineux, sa plus grande dimension, dans le sens de la longueur, est de $125 \mu$. Distances du centre de la ventouse au cloaque et à l'extrémité distale égales respectivement à $500 \mu$ et $820 \mu$.

Anus à $320 \mu$ de l'extrémité postérieure.

La queue porte 10 paires de papilles caudales disposées comme suit: arrière ;

- 4 paires de papilles préanales, 1 paire en avant de la ventouse et 3 paires en

- 6 paires de papilles postanales, 4 paires ventro-latérales dans le prolongement des papilles préanales, disposées en deux groupes, un groupe de 2 paires immédiatement en arrière du cloaque et un groupe de 2 paires proche de l'extrémité postérieure; et 2 paires latérales situées entre ces deux groupes (fig. II, A, B, C).

Pas de gubernaculum.

2 spicules subégaux mesurant $245 \mu$ (spicule gauche) et $250 \mu$ (spicule droit) sur, respectivement, $25 \mu$ et $30 \mu$ de large, courts, légèrement courbés avec un manche bien chitinisé et une extrémité distale plus frêle, faiblement ailée, bilobée (fig. II, D).

\section{Description de la femelle.}

Corps long de $11 \mathrm{~mm}$ et large de $230 \mu$ au niveau de l'intestin antérieur. Toutes les dimensions données se rapportent à cet exemplaire. 


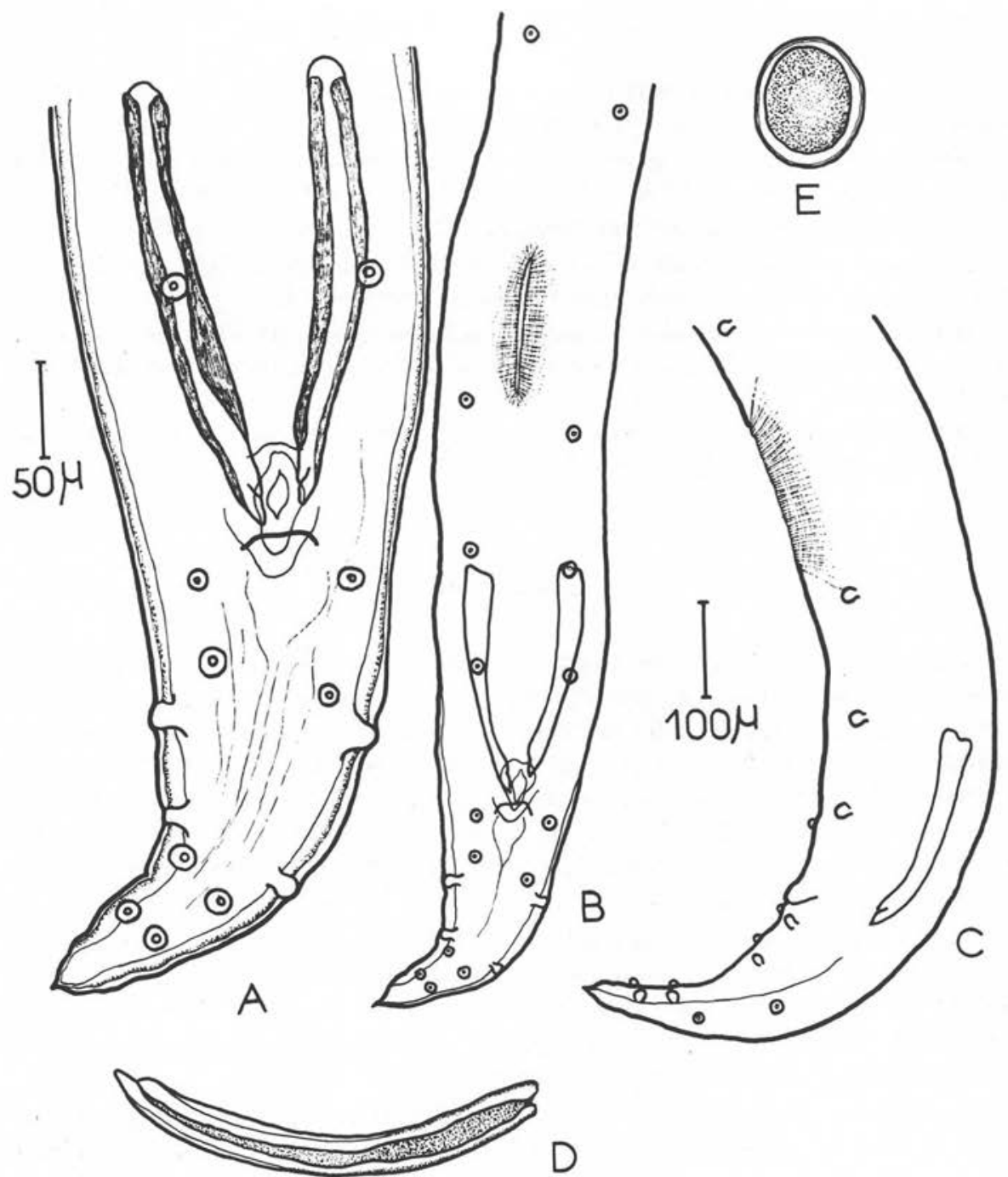

FIG. II. - Quimperia lanceolata, Gendre, 1926
A : région postérieure du mâle, vue ventrale.
B : queue du mâle, vue ventrale.
C: queue du mâle, vue latérale.
D: spicule dissśqué.
$\mathrm{E}$ : œuf.

(A, D, E : échelle $50 \mu ; \mathrm{B}, \mathrm{C}$ : échelle 100 «) 
Cuticule épaisse de 3 à $4 \mu$.

Pore excréteur, anneau nerveux et déirides respectivement situés à $325 \mu, 380 \mu$ et $900 \mu$ de l'apex.

Ailes latérales longues de $880 \mu$ sur $35 \mu$ de large, débutant à $60 \mu$ de l'extrémité antérieure et se terminant à $940 \mu$ au niveau des déirides.

Première et deuxième partie de l'œsophage mesurant respectivement $400 \mu$ de long sur $50 \mu$ de large et $725 \mu$ sur $140 \mu$. Longueur totale de l'œesophage égale à $1.125 \mu$.

Queue terminée far une fine pointe caudale à $280 \mu$ de l'anus (fig. I, E).

Femelle ovipare, vulve saillante, en situation post-équatoriale à $7,875 \mathrm{~mm}$ de l'apex. Rapport longueur du corps sur distance de la vulve à l'apex égal à 1,4 .

Ovéjecteur court et fortement musculaire, mesurant $210 \mu$ sur $65 \mu$ de diamètre et dirigé vers l'avant du corps. 2 utérus divergents en arrière de l'ovéjecteur l'un vers l'avant, l'autre vers l'arrière du corps.

CEufs ellipsoïdes à sub-sphériques, à coque très mince, non embryonnés et mesurant $65 \mu$ sur $55 \mu$ en moyenne (fig. II, E).

\section{Discussion}

La description originale de Gendre (1926) est suffisamment précise pour nous permettre d'identifier nos Nématodes à Quimperia lanceolata. En effet, nous ne constatons pratiquement aucune différence entre nos spécimens et les siens. Les dimensions générales sont semblables bien que nos exemplaires soient légèrement plus grands; la structure buccale, celle de l'œsophage, l'aspect lancéolé du corps dû à la présence des ailes latérales antérieures, la situation de la vulve et les dimensions des œufs chez la femelle; la longueur des spicules, le nombre et la disposition des papilles caudales chez le mâle, sont identiques.

Notre étude complète la description morphologique de l'espèce notamment pour ce qui concerne la structure apicale, la position du pore excréteur et surtout la morphologie des spicules que nous avons pu disséquer; elle confirme l'absence de gubernaculum ; caractères que Gendre n'avait pas pu observer sur ses exemplaires \& devenus noirs par suite d'une mauvaise conservation» (Gendre, 1926).

Par ailleurs, au sujet de l'hôte, il est vraisemblable que le poisson chez qui a été trouvé en Guinée Quimperia lanceolata ( Poisson Acanthoptérygien non déterminé ») appartient également au genre Ctenopoma Peters, 1844, Anabantidae dont l'espèce unique en Afrique occidentale est C. kingsleyae Günther, 1896 (Daget, 1954).

Ce poisson très commun en Guinée est fréquemment pêché à Sangalkam, région qui est justement considérée par sa flore et sa faune comme un îlot de région « guinéenne » au Sénégal. De plus, il semble exister une étroite spécificité parasitaire entre Quimperia lanceolata et Ctenopoma kingsleyae car, dans les marigots de Sangalkam où coexistent intimement Ctenopoma, Tilapia sp. (Cichlidae) et Clarias sp. (Clariidae) seuls les Ctenopoma hébergent constamment Quimperia lanceolata. 


\section{Bibliographie}

Chabaud (A.-G.), Campana-Rouget (Y.) et Brygoo (E.-R.), 1959. - Les Nématodes Seuratoidea, nov. sup. fam. et l'origine des Spirurida. C.R. Acad. Sc. Paris, CCXLVIII, 1449-1451.

—, —, et -, 1960. — Les Nématodes Seuratoidea. Ann. parasit. hum. comp., XXXV, (3) 316-346.

Daget (J.), 1954. - Les poissons du Niger supérieur. Mémoires de l'Institut français d'Afrique Noire, $\mathrm{n}^{\circ}$ 36. I.F.A.N., Dakar, 391 pages.

Gendre (E.), 1926. - Sur un Nématode nouveau, parasite de poisson. Bull. Soc. Path. exot., XIX, 798-802. 